\title{
Research of Anti-Cancer Components in Traditional Chinese Medicine on Hollow Fibre Cell Fishing and Hollow Fibre Liquid Phase Microextraction
}

\author{
Feixue Li, Xuan Chen, Yanli Xu, Xiaohong Bai* \\ School of Pharmacy, Shanxi Medical University, Taiyuan, China \\ Email: *bxh246@sxmu.edu.cn
}

How to cite this paper: Li, F.X., Chen, X., Xu, Y.L. and Bai, X.H. (2016) Research of Anti-Cancer Components in Traditional Chinese Medicine on Hollow Fibre Cell Fishing and Hollow Fibre Liquid Phase Microextraction. American Journal of Analytical Chemistry, 7, 696-711.

http://dx.doi.org/10.4236/ajac.2016.710063

Received: August 22, 2016

Accepted: October 10, 2016

Published: October 13, 2016

Copyright $\odot 2016$ by authors and Scientific Research Publishing Inc. This work is licensed under the Creative Commons Attribution International License (CC BY 4.0).

http://creativecommons.org/licenses/by/4.0/

\begin{abstract}
Hollow fibre cell fishing with HPLC (HFCF-HPLC) based on the human ovarian cancer cell line SKOV-3, human renal tubular cell line ACHN or hepatoma cell line HepG-2 was employed to screen active groups of coumarin and volatile oil in Radix angelicae sinensis, Radix angelicae dahuricae and Fructus citri sarcodactylis. Simultaneously, hollow fibre liquid phase microextraction with HPLC (HFLPME-HPLC) was conducted to enrich and determine the contents of active components in the same sample solution. Before application, for HFCF-HPLC, cells growth states and survival rates on the fibre, effect of ethanol concentration in the extract of samples on cell survival rates, non-specific binding between fibre active centres and the target components, positive and negative controls and repeatabilities were validated; for HFLPME, extraction solvent, sample phase $\mathrm{pH}$, agitation speed, extraction time and sample phase volume were investigated. Many active components were screened from three medicines. Some of them, such as scoparone, psoralen, bergapten, oxypeucedanin, imperatorin, ligustilide, were identified by MS. The target fishing factors of active components and the cell apoptosis rates of three cells under the medicines effect were researched. The binding sites of active groups on HepG-2 cells were preliminarily determined. The results demonstrated that HFCF-HPLC, coupled with HFLPME-HPLC, is a simple and universal approach to find bioactive components at the cellular level, determine their content and research traditional Chinese medicines (TCMs) entirety effect of multi-component and multi-target. The approach may provide us a new and good solution to clarify the material basis of anti-cancer effect and conduct personalized quality control for the components associated with efficacy in TCMs.
\end{abstract}




\section{Keywords}

Hollow Fibre Cell Fishing, Liquid Phase Microextraction, Identification, Quantification, Cancer Cell, Activity Screening

\section{Introduction}

Traditional Chinese medicines (TCMs) of Radix angelicae sinensis, Radix angelicae dahuricae and Fructus citri sarcodactylis mainly contain coumarin and volatile oil. Coumarin, for instance, psoralen, bergapten, isobergapten, oxypeucedanin, imperatorin and isoimperatorin have anti-oxidant [1] and anti-cancer [1]-[3] effects. Volatile oil, such as ligustilide has the properties of anti-cancer [4], anti-platelet aggregation and anti-thrombotic [5]. It is well known that the main effect characteristic of TCMs is the synergy effect of multi-components, multi-target rather than a single component, a single target. So, it is necessary to make clearly the anti-cancer material basis of multicomponent and multi-target synergy effect in three TCMs.

Traditional activity screening method [6] is time-consuming with high labor intensity and unlikely to simultaneously screen and quantify active components in the same sample solution. Recently, our research team have proposed and developed a procedure at the cellular level to research the active component groups in TCMs, i.e. hollow fibre cell fishing with high performance liquid chromatography (HFCF-HPLC) [7]-[9]. In HFCF, the fibre seeded cancer cells was functioned as an activity research platform to screen and identify the anticancer active components in TCM. Soon after, we used respectively hollow fibres filled with the cell membrane, cell organelle [10]-[12] or the receptors [11] [12] respectively to research the binding sites and targets of the active component groups combined with cells in TCMs preliminarily. But the research of simultaneous screening and quantifying active components in the same sample solution has not been published.

Hollow fibre liquid phase microextraction (HFLPME) is a sample preparation procedure. In HFLPME, the analytes are extracted and enriched by passive diffusion from sample phase into extraction solvent that immobilized in pores and filled in the hollow fibre lumen [13]. Up to now, HFLPME [14]-[16] coupled with HPLC, has been applied for determining various of active components in TCMs because its simplicity, less solvent consumption, low cost, high enrichment and excellent sample clean-up.

In the paper, HFCF, based on the human ovarian cancer cell line SKOV-3, human renal tubular cell line ACHN or hepatoma cell line HepG-2, coupled with HPLC, was employed to screen and identify anti-cancer components from Radix angelicae sinensis, Radix angelicae dahuricae and Fructus citri sarcodactylis. Simultaneously, HFLPME with HPLC was used to determine the contents of the identified active components in the same sample. In the experiment, some structures of coumarin and volatile oil active components, for example scoparone, psoralen, bergapten, oxypeucedanin, imperatorin and ligustilide, in three TCMs have been identified by retention time or mass spectra. 
The target fishing factors of active components and the cell apoptosiss rates of three cells under the TCMs effect were determined. The binding sites, cell membrane or cell organelle, of the active groups on HepG-2 cells were preliminarily researched and discussed. The research results verified the TCMs effect characteristic of the multi-component and multi-target further. This study aimed to establish a simple, quick procedure and employ it to identify and quantify simultaneously the anti-cancer active components of coumarin and volatile oil in the same sample solution, meanwhile, prove TCM effect of multi-component and multi-target and conduct personalized quality control.

\section{Materials and Methods}

\subsection{Instruments and Apparatus}

The following instruments were utilized: liquid chromatograph (1200 series) equipped with two pumps (G1311A), a thermostat (G1316A), and a detector (UV) (Agilent Technologies, Palo Alto, CA, USA); Thermal FE Environment Scanning Electron Microscope (SEM, JSM-7001F, JEOL, Japan); $\mathrm{CO}_{2}$ incubator (Thermo Fisher Scientific, Waltham, MA, USA); FACS Calibur flow cytometer (Becton, Dickinson and Company, Franklin, NJ, USA); micr OTOF-Q III high-performance Electrospray Ionization-time of Flight-grade rod LC/MS/MS Tandem mass spectrometer (Bruker, Germany); high-speed refrigerated centrifuge (TGL 16M, Changsha, China), Ronghua Group 85 2 magnetic heater with stirrer (Jiangsu, China) and $8 \mathrm{~mm} \times 4 \mathrm{~mm}$ stirring bar.

\subsection{Chemical Standards}

The analytical reference substances for umbelliferone (batch number: MUST-14120715, purity $\geq 99.71 \%$ ), scoparone (batch number: MUST-15041013, purity $\geq 99.96 \%$ ), psoralen (batch number: MUST-15012215, purity $\geq 99.99 \%$ ), bergapten (batch number: A100525, purity $\geq 98 \%$ ), isobergapten (batch number: A0612B, purity $\geq 98 \%$ ), oxypeucedanin (batch number: A71223, purity $\geq 98 \%$ ), imperatorin (batch number: A0011, purity $\geq 98 \%$ ), ligustilide (batch number: MUST-14122610, purity $\geq 98 \%$ ), isoimperatorin (batch number: A0012, purity $\geq 98 \%$ ), columbianadin (batch number: MUST15032413, purity $\geq 98 \%$ ), were obtained from Chengdu MUST Bio-Technology Co., Ltd. Radix angelicae sinensis (batch number: 20150823, producing area: GanSu, China), Radix angelicae dahuricae (batch number: 20150806, producing area: AnHui, China) and Fructus citri sarcodactylis (batch number: 20150806, producing area: Guang Dong, China) were purchased from Tongrentang drugstore (Taiyuan, Shanxi, China) and authenticated by associate professor (Y. E. Bai) of Chinese Pharmacy in the School of Pharmacy, Shanxi Medical University.

\subsection{Material and Reagent}

The polyvinylidene fluoride fibre (PVDF) was purchased from Tianjin Motianmo Engineering Co., Ltd (Tianjin, China). HPLC-grade acetonitrile and methanol were purchased from Tianjin Siyou Chemical (Tianjin, China). SKOV-3, ACHN, HepG-2 were 
procured from Wuhan Boster Bio-Engineering Co., Ltd. (Wuhan, China). All medium (Wuhan Boster Bio-Engineering Co., Ltd., Wuhan, China), foetal bovine serum (Zhejiang, China), trypsin solution and penicillin-Streptomycin (Beijing Solarbio Science \& Technology Co., Ltd., Beijing, China) were used in cell culture. Other reagents were of analytical grade. Double-distilled water was used throughout the whole study.

\subsection{Preparation of Reference and Sample Solutions}

Reference solution. Prepare standard stock solutions in methanol contain $200 \mu \mathrm{g} / \mathrm{mL}$ bergapten, isobergapten and oxypeucedanin, $400 \mu \mathrm{g} / \mathrm{mL}$ umbelliferone, scoparone, psoralen, imperatorin and columbianadin and $2 \mu \mathrm{g} / \mathrm{mL}$ ligustilide and isoimperatorin. The mixed working solution of ten components was prepared daily by diluting the previous stock solutions with double distilled water. All of the solutions were stored at approximately $4^{\circ} \mathrm{C}$.

Preparation of water decoctions. The sample of powdered Radix angelicae sinensis (20.0 g), Radix angelicae dahuricae (15.0 g) and Fructus citri sarcodactylis (15.0 g) were respectively dissolved in double distilled water of $200 \mathrm{~mL}$, soaked and slightly boiled twice for $30 \mathrm{~min}$ each time. The weight loss was compensated with corresponding volume of double distilled water after the solution was cooled to room temperature. Centrifuge and take the supernatant. These solutions were stored at $4{ }^{\circ} \mathrm{C}$.

Preparation of ethanol extracts. The sample of powdered Radix angelicae sinensis (10.0 g), Radix angelicae dahuricae (10.0 g) and Fructus citri sarcodactylis (10.0 g) were soaked respectively in a mixture solution of ethanol and water of $80 \mathrm{~mL}(v: V=85: 15)$ [17], $80 \mathrm{~mL}(v \cdot V=75: 25)$ [18], 50mL ( $v: v=60: 40)$ [19] followed by ultrasonic wave extraction for $60 \mathrm{~min}$. The rest of the operation was the same as above.

\subsection{Preparation of Fibre}

Prior to use, the hollow fibre was ultrasonically cleaned in acetone, methanol and water for 15 min successively, and then dried in air.

Preparation of the fibre seeded cells. The SKOV-3, ACHN and HepG-2 cell lines were maintained respectively in McCoy's 5A, IMDM and DMEM medium and were placed in a humidified incubator with $5 \% \mathrm{CO}_{2}$ at $37^{\circ} \mathrm{C}$. After $24 \mathrm{~h}$ to $48 \mathrm{~h}$, the logarithmic phase of cells were obtained. These cells were digested with trypsin at $37^{\circ} \mathrm{C}$ for $3 \mathrm{~min}$. The trypsinized cells were suspended in the nutrient solution, and then centrifuged at $1000 \mathrm{rpm}$ for $5 \mathrm{~min}$. The supernatant fluid was discarded and $500 \mu \mathrm{L}$ of nutrient solution was added. The cell suspensions were drawn into a syringe tube without needle and then the needle was installed on the syringe tube. Afterwards the hollow fibers were placed in the culture flask, the cells in the syringe were injected into the fibres until another port began releasing the liquid. The nutrient solution was added to the culture flask and cultivated for $20 \mathrm{~h}$ [12]. The fibre seeded cells was used for activity screening by HFCF within 6 hours [9] after cultivation.

Preparation of the fibre filled with cell membrane and organelle. The cultured HepG-2 cell $\left(1 \times 10^{7}\right)$ were washed with phosphate buffer solution (PBS) for three times 
then centrifuged (1000 rpm, $10 \mathrm{~min})$. Tris- $\mathrm{HCl}(\mathrm{pH} 7.4)$ was added to produce a cell suspension, which was ruptured by sonication $(30 \mathrm{~min})$ immediately. After the resulting clarified liquid was centrifugated $\left(1000 \times g, 10 \mathrm{~min}, 4^{\circ} \mathrm{C}\right)$, the pellet and supernate were collected, respectively. The pellet was cell organelle and the supernate was centrifugated $\left(10,000 \times g, 20 \mathrm{~min}, 4^{\circ} \mathrm{C}\right)$ to give the cell membrane precipitate. The cell membrane and cell organelle suspensions were prepared by $500 \mu \mathrm{L}$ nutrient solution and used immediately. The above-mentioned suspensions were injected into fibre lumen by microsyringes respectively [10]. The fibres filled with cell membrane or cell organelle was used for activity screening by HFCF within 6 hours [9] after cultivation.

Preparation of fibre filled with solvent. $7.0 \mathrm{~cm}$ hollow fibres were immersed in the mixed extraction solvent of chlorobenzene and hexanol ( $v: v=5: 5)$, until its lumen and wall pores were filled with the extraction solvent completely. The fibres were removed by a tweezer from the extraction solvent, and the excess solvent outside the fibres was rubbed off with an absorbent paper. The fibres filled with extraction solvent were used immediately for HFLPME.

\subsection{Chromatographic and Spectroscopic Conditions}

The C 18 column $(250 \mathrm{~mm} \times 4.6 \mathrm{~mm}, 5 \mu \mathrm{m}$, Agilent Technologies $)$ was maintained at $37^{\circ} \mathrm{C}$ during the whole process. To optimize the separation of the coumarin and volatile oil in the extract, the gradient conditions of the binary mobile phase as follow: A (acetonitrile) and $\mathrm{B}$ (water) [7]: 0 to $3 \mathrm{~min}, 30 \% \mathrm{~A} ; 3$ to $5 \mathrm{~min}, 30 \%-60 \% \mathrm{~A} ; 5$ to $8 \mathrm{~min}$, $60 \%-70 \% \mathrm{~A} ; 8$ to $11 \mathrm{~min}, 70 \%-60 \% \mathrm{~A} ; 11$ to $14 \mathrm{~min}, 60 \%-60 \% \mathrm{~A} ; 14$ to $16 \mathrm{~min}, 60 \%-$ $30 \% \mathrm{~A}$. The detection wavelength was set at $324 \mathrm{~nm}$. The flow rate was $1.0 \mathrm{~mL} / \mathrm{min}$.

\subsection{Test Procedure}

The HFCF-HPLC (Figure 1A) and HFLPME-HPLC (Figure 1B) procedures as follow. In the experiment, $7.0 \mathrm{~mL}$ of the solution (mixed working solution, TCM water decoction or water solution containing $10 \%$ TCM ethanol extract) and a magnetic stirrer bar were placed in a $10 \mathrm{~mL}$ sample vial fixed on the magnetic stirrer. A few $7.0 \mathrm{~cm}$ hollow fibres seeded cells, filled with cell membranes, organelles and mixed extraction solvent were sealed with a cotton thread tied on both ends respectively, bent into a U-shape, and then inserted simultaneously into the same sample vial. The active components in TCM were screened and extracted at $600 \mathrm{rpm}$ and $37^{\circ} \mathrm{C}$. After 60 min extraction, the fibres containing mixed extraction solvent were removed from the vial, the seals at both ends were cut, the active components enhanced by extraction solvent were eluted by 40 $\mu \mathrm{L}$ of methanol and transferred into Eppendorf (EP) tube, the extractant of $20 \mu \mathrm{L}$ was injected into HPLC system for quantitative analysis; After $3 \mathrm{~h}$ screening, other fibres were removed and cut their ends seals, the active components acted on biological target were dissociated by $40 \mu \mathrm{L}$ of methanol and transferred respectively into EP tube, then centrifuged at 10,000 rpm for $20 \mathrm{~min}$. The supernatant fluid of $20 \mu \mathrm{L}$ was injected into HPLC system for qualitative analysis. The fibre filled with nutrient solutions and blank control screenings were also performed. Each process was performed thrice. 


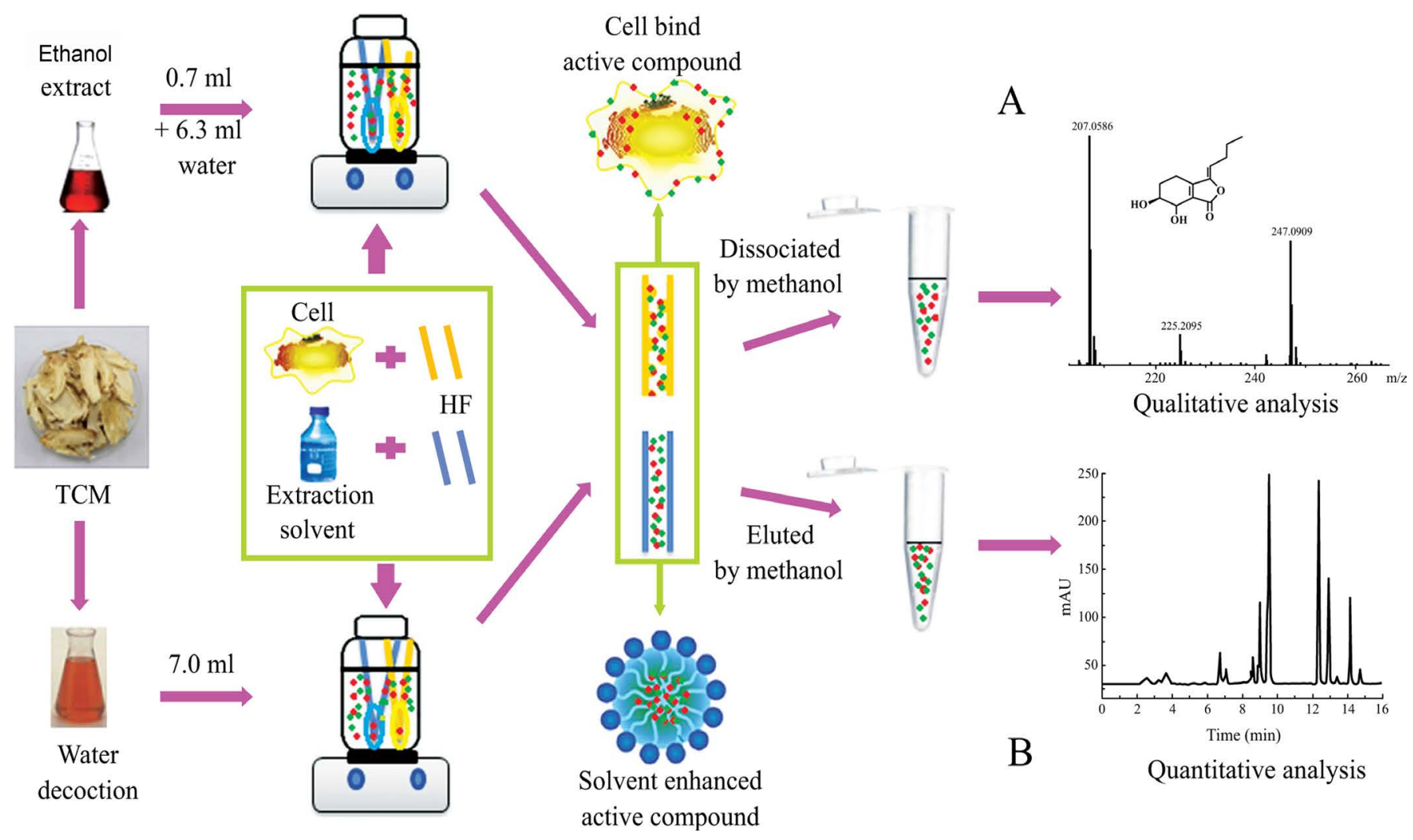

Figure 1. The schematic diagrams of HFCF-HPLC (A) and HFLPME-HPLC (B) process and mechanism.

\section{Results}

\subsection{HFCF-HPLC Qualitative Analysis Procedure}

\subsubsection{Selection of Screening Condition}

Cell growth state on the inner wall of fibre. In order to certain the cell growth state on the inner wall of hollow fibre, we compared the SEM images of the nutrient solution and cells seeded on the inner wall of fibre. We can see from Figure 2 that the micrograph of the inner surface of fibre seeded nutrition solution (Figure 2A) appears smooth and without any additives. The micrographs of the fibre internal surface seeded HepG-2 (Figure 2B) and ACHN cells (Figure 2C) have obviously knob, asperities and tentacles, which indicate that the cells have been seeded and adhered onto the internal surface tightly.

Survival rate before screening. Cells should healthily grow and maintain viable in the screening process. The survival rates of SKOV-3, ACHN and HepG-2 cells seeded on the internal surface of fibre were determined by flow cytometry. Survival rate was defined as the percentage of the total normal (annexinV-negative, PI-negative) cells. The results showed that the survival rates of the cells seeded fibre before screening were $86.88 \%$, 96.45\%, 90.1\%, respectively.

Effect of ethanol concentration on HepG-2 cells survival rate. In order to eliminate ethanol effect on the cells seeded on fibre internal surface, we tested the effect of 


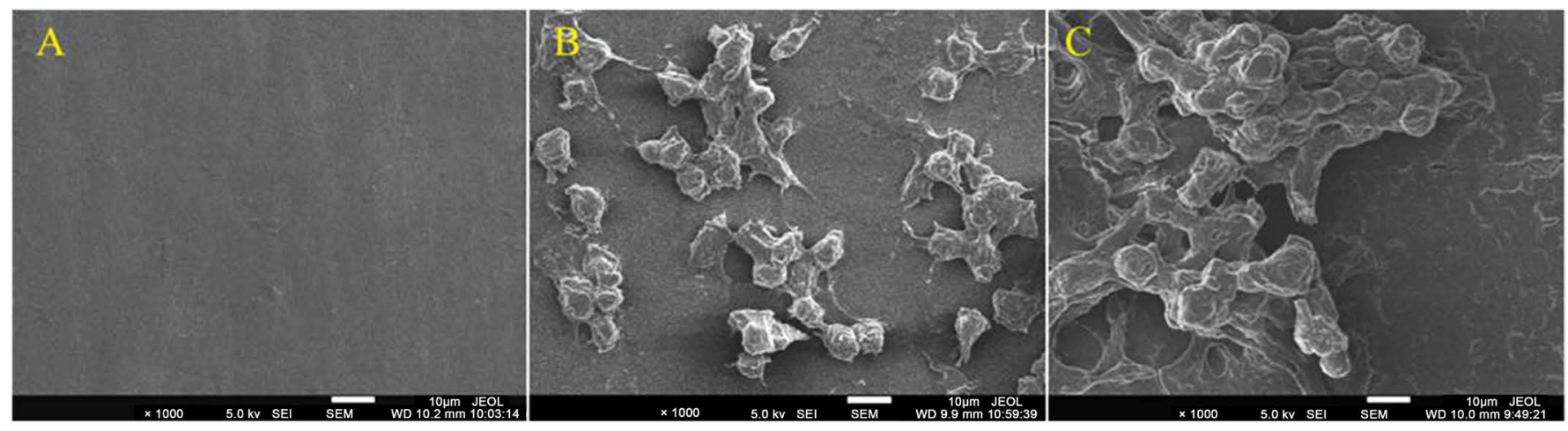

Figure 2. SEM micrographs of the internal surface of the fibre seeded nutrition solution (A), HepG-2 (B) and ACHN (C) cells at $5 \mathrm{kV}$ $\times 1000$.

ethanol black solutions of different concentrations $(0 \%, 5 \%, 7 \%, 10 \%, v / v)$ on the survival rates of HepG-2 cells in the screening process. The results (Figure 3 ) show that the survival rate of HepG-2 cells before screening is $90.1 \%$ (Figure 3A), after screening ethanol black solutions of different concentrations $(0 \%, 5 \%, 7 \%, 10 \%, \mathrm{~V} / \mathrm{V})$, the survival rates of HepG-2 cells are $87.58 \%$ (Figure 3B), 84.29\% (Figure 3C), 84.24\% (Figure 3D), $83.86 \%$ (Figure 3E). There is no significant difference in HepG-2 survival rates when ethanol concentration below $10 \%(v / v)$ in the sample solution. Therefore, the cell seeded fibre could be used for the screening actives in TCM ethanol extract (ethanol concentration below $10 \%)$.

\subsubsection{Validation of the Method}

Binding between fibre activity centre and active component. The chromatographic behaviors of the active components were investigated after being screened and isolated. The hollow fibre with nutrient solution screening sample in Line b of Figure 4, the chromatographic peak area and the number of the component screened are smaller and less than those obtained by hollow fibres with cells screening sample in Line $c$ of Figure 4. The results indicated that the main interaction mechanism of HFCF was the binding between cells and bioactive components rather than the binding between activity centres of hollow fibre and bioactive components.

Positive and negative control. In previous work, our group selected paclitaxel [11], a broad-spectrum anticancer medicine, as positive control and screened by HFCFHPLC based on ACHN and HepG-2 cells. Tamoxifen as positive and indomethacin as negative control were did by HFCF-HPLC on MCF-7 cells [10]. The determination results indicated that the $C F F$ of paclitaxel on ACHN and HepG-2 were 2.0, 2.4, respectively, and tamoxifen's $C F F_{\mathrm{MCF}-7}^{\mathrm{wa}}=5.5$, indomethacin's $C F F_{\mathrm{MCF}-7}^{\mathrm{wa}}=0$ on MCF-7.

Repeatability. In this experiment, we investigated the repeatability of the retention times $\left(t_{\mathrm{R}} \mathrm{s}\right)$ and the relative peak areas (RPAs) of the components screened by HFCF-HPLC. The relative standard deviation (RSDs) of the $t_{\mathrm{R}} \mathrm{s}$ and the RPAs in water extract are $<0.60 \%$ and $<9.28 \%$, in ethanol extract are $<0.80 \%$ and $<4.07 \%$, respectively. The results (Table 1) indicate that the HFCF-HPLC method used to screen active components from Radix angelicae sinensis, Radix angelicae dahuricae, Fructus citri 

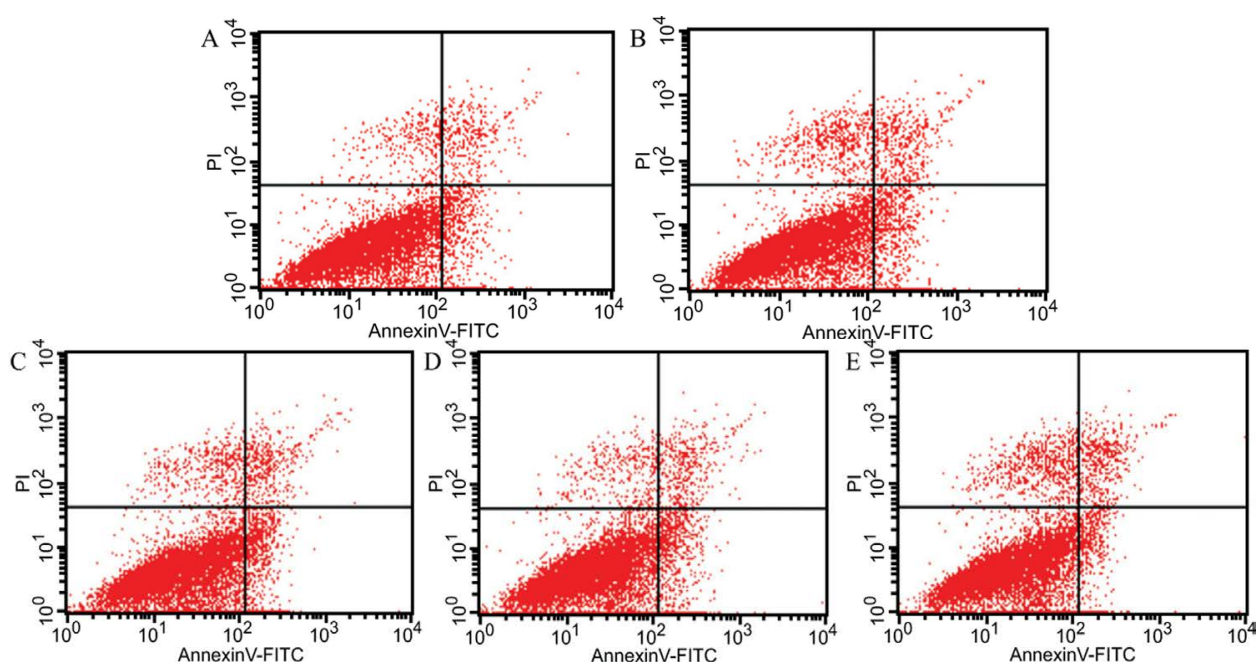

Figure 3. The flow cytometry results of HepG-2 cells seeded on HF (A) and after the cells screening blank solution with different ethanol concentrations as follow: $0 \%(B), 5 \%(C), 7 \%(D)$, $10 \%(\mathrm{E})(\mathrm{v} / \mathrm{v})$.

sarcodactylis has good repeatability.

\subsection{HFLPME Quantitative Analysis Procedure}

\subsubsection{Optimization of HFLPME}

To ensure the reliability and sensitivity of results under the activity screening conditions $\left(7.0 \mathrm{~mL}\right.$ of sample solution $(\mathrm{pH}=7)$ was extracted at $600 \mathrm{rpm}$ and $37^{\circ} \mathrm{C}$, the result showed in electronic supplementary information), we investigated and optimized the effects of extraction solvent (Figure 5) and extraction time in HFLPME-HPLC on the enrichment factors $(E F s)$ of the target analytes. We find that, in Figure 5A, (1) no target component is extracted by pentanol; (2) nine components (except psoralen) can be enriched by hexanol and their EFs are high; (3) ten components can be enriched by chlorobenzene, but their $E F$ s are low. So the mixed solvents, as extraction solvent, of hexanol and chlorobenzene at different proportions were chosen and examined in the experiment. In Figure 5B, when the proportion is 5:5 ( $\mathrm{v} \cdot \mathrm{V})$, all target components, in particular psoralen and bergapten, can be simultaneously extracted and enriched and their enrichment factors are higher. Therefore, the mixed solvent of hexanol and chlorobenzene at the proportion of 5:5 $(\mathrm{v}: \mathrm{V})$ was selected in later experiments.

Different extraction times $(20,30,50,60,70 \mathrm{~min})$ also were investigated. The result illustrated that $60 \mathrm{~min}$ provided the highest enrichment factors. Therefore, $60 \mathrm{~min}$ was selected for the succeeding experiments.

\subsubsection{Method Validation}

Linearity and limit of detection. The calibration curves were obtained by plotting peak areas $(A s)$ against the concentrations of the ten analytes $(C s)$. The limits of detection (LODs) and the limits of quantification (LOQs) were obtained by determining the concentration of each analyte under the signal of $S / N=3$ and $S / N=10$ [20]. The linear ranges, correlation coefficients, LODs and LOQs are listed in the Table 2. 

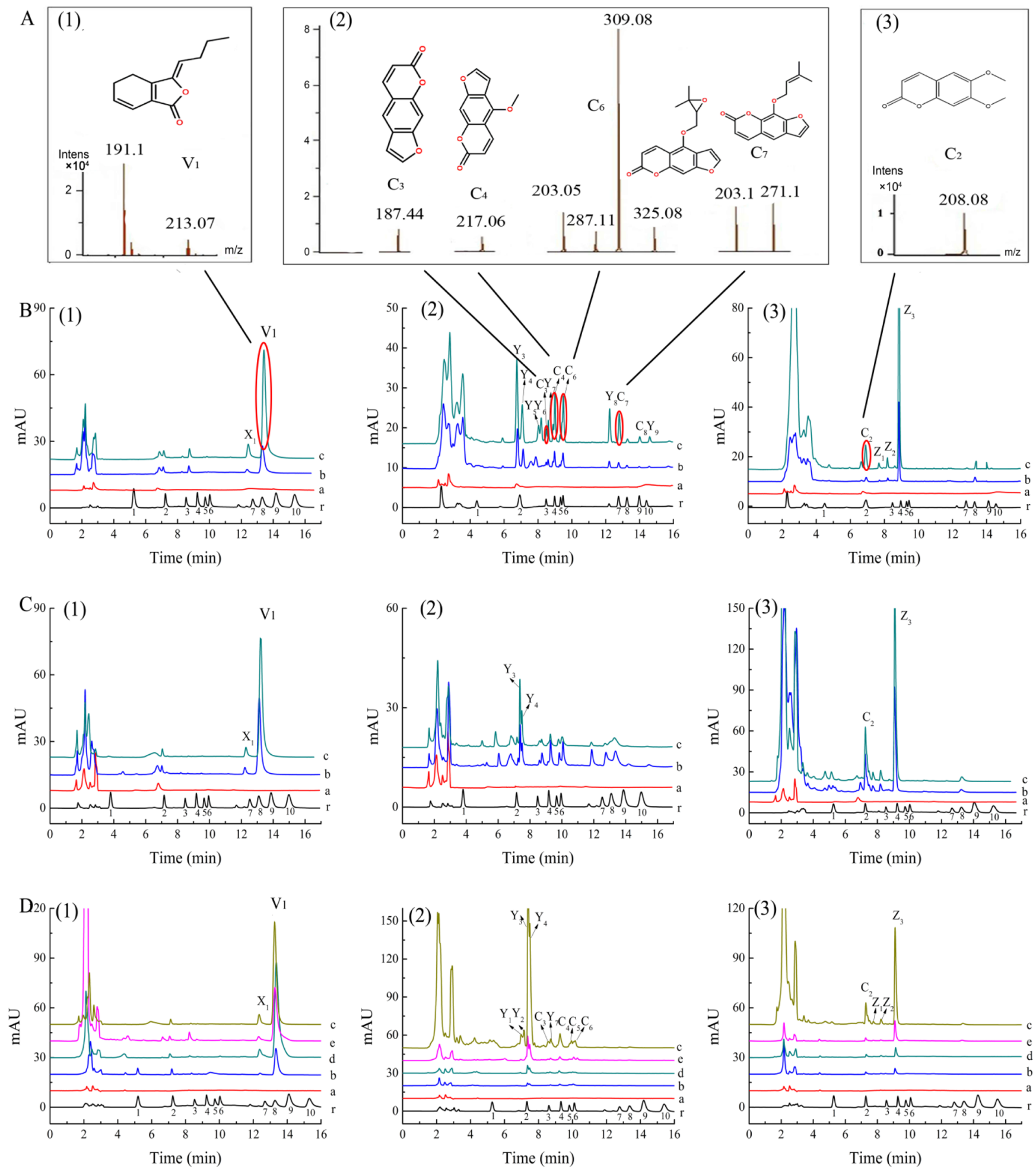

Figure 4. Chromatograms of the active components in total water decoction of the three TCMs (A) LC/MS of SKOV-3 cell, LC/UV of (B) SKOV-3 (C) ACHN and (D) HepG-2 cells screenning in Radix angelicae sinensis (1) radix angelicae dahuricae (2) and fructus citri sarcodactylis (3) reference substance (r); blank (a); nutrient solution screening (b); cell screening (c); cell organelle screening (d); cell membrane (e) screening $\mathrm{C}_{1}$. umbelliferone; $\mathrm{C}_{2}$. scoparone; $\mathrm{C}_{3}$. psoralen; $\mathrm{C}_{4}$. bergapten; $\mathrm{C}_{5}$. isobergapten; $\mathrm{C}_{6}$. oxypeucedanin; $\mathrm{C}_{7}$. imperatorin; $\mathrm{V}_{1}$. ligustilide; $\mathrm{C}_{8}$. isoimperatorin; $\mathrm{C}_{9}$. columbianadin; $\mathrm{X}_{1}, \mathrm{Y}_{1-9}, \mathrm{Z}_{1-3}$, unknown active components. 
Table 1. The precisions of screening results for active components in TCM ethanol extract and water decoction using HFCF-HPLC.

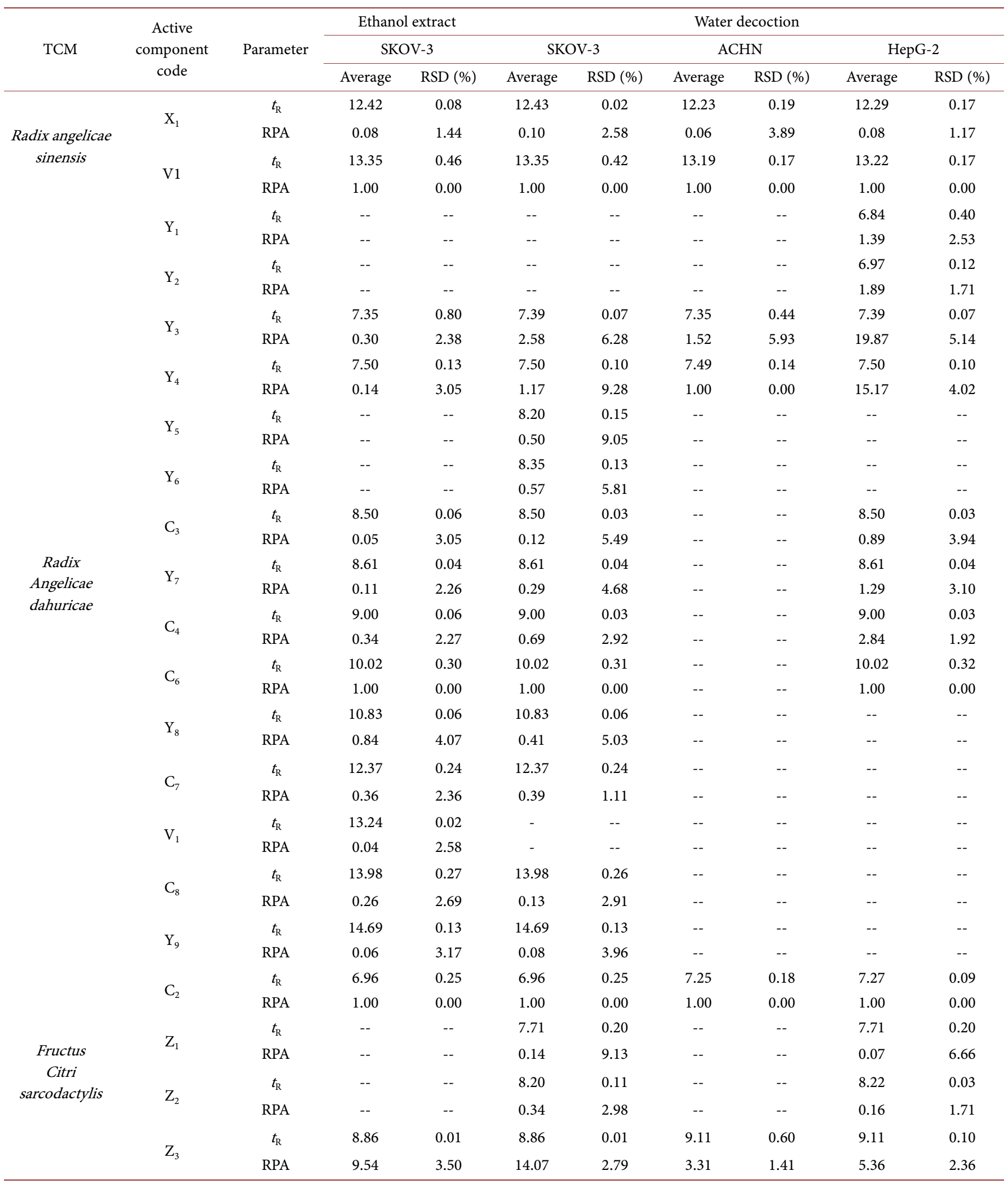

“--": the active component was not screened out. 

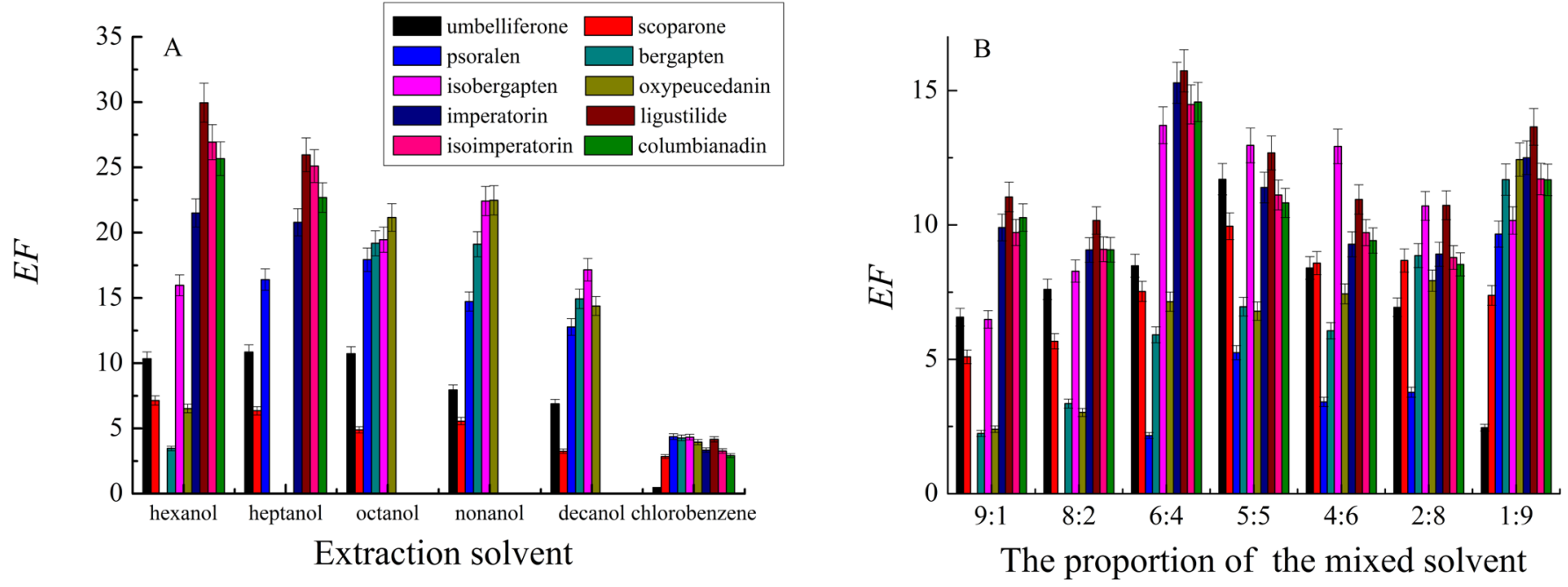

Figure 5. Effect of extraction solvent and the proportion of the mixed solvent on EF of analyte extraction condition: concentration of analytes, $1.33 \mu \mathrm{g} / \mathrm{mL}$ for umbelliferone and scoparone, $2 \mu \mathrm{g} / \mathrm{mL}$ for psoralen, bergapten, isobergapten, oxypeucedanin and ligustilide, 4 $\mu \mathrm{g} / \mathrm{mL}$ for imperatorin and columbianadin, $5 \mu \mathrm{g} / \mathrm{mL}$ for isoimperatorin; sample solution $\mathrm{pH}$, 7; extraction time, $60 \mathrm{~min}$; stirring rate, 600 rpm; volume of sample phase, $7.0 \mathrm{~mL}(\mathrm{n}=3)$.

Table 2. The data of method validation of the HFLPME coupled with HPLC.

\begin{tabular}{|c|c|c|c|c|c|}
\hline Analyte & $\begin{array}{l}\text { Regression } \\
\text { equation }\end{array}$ & $\begin{array}{c}\text { Correlation coefficient } \\
\qquad \mathrm{R}^{2}\end{array}$ & $\begin{array}{l}\text { Linear range } \\
\left(\mathrm{ng} \cdot \mathrm{mL}^{-1}\right)\end{array}$ & $\begin{array}{l}\text { Detection limit } \\
\left(\mathrm{ng} \cdot \mathrm{mL}^{-1}\right)\end{array}$ & $\begin{array}{c}\text { Enrichment factor } \\
E F\end{array}$ \\
\hline Umbelliferone & $Y=2569.7 X+9.3102$ & 0.9987 & $0.769 \sim 769.2$ & 0.154 & 29.3 \\
\hline Psoralen & $Y=366.07 X-1.5767$ & 0.9961 & $0.154 \sim 769.2$ & 0.077 & 10.6 \\
\hline Bergapten & $Y=1092.7 X-3.9867$ & 0.9957 & $0.769 \sim 769.2$ & 0.154 & 15.7 \\
\hline Oxypeucedanin & $Y=774.77 X+5.6622$ & 0.9945 & $1.538 \sim 1538$ & 0.308 & 29.0 \\
\hline Imperatorin & $Y=1061.3 X-34.311$ & 0.9949 & $3.077 \sim 3077$ & 0.616 & 60.0 \\
\hline Ligustilide & $Y=361.88 X-50.594$ & 0.9962 & $15.38 \sim 15385$ & 3.077 & 56.8 \\
\hline Isoimperatorin & $Y=2308.3 X-65.04$ & 0.9966 & $7.692 \sim 3846$ & 3.846 & 55.0 \\
\hline Columbianadin & $Y=2548.1 X-51.401$ & 0.9963 & $6.154 \sim 3077$ & 3.077 & 65.5 \\
\hline
\end{tabular}

Precision. The precision of this method, which was expressed as relative standard deviation (RSD), was calculated by using three replicate determinations of standard solutions at their concentrations $(0.015 \mu \mathrm{g} / \mathrm{mL}$ for umbelliferone, scoparone, psoralen and bergapten, $0.031 \mu \mathrm{g} / \mathrm{mL}$ for isobergapten and oxypeucedanin, $0.062 \mu \mathrm{g} / \mathrm{mL}$ for imperatorin and columbianadin, $0.307 \mu \mathrm{g} / \mathrm{mL}$ for ligustilide, $0.077 \mu \mathrm{g} / \mathrm{mL}$ for isoimperatorin) within one day (intra-day precision) and three replicates over three days (inter-day precision) [21]. The RSDs for the intra-day and inter-day precision ranged from $1.4 \%$ to $9.0 \%$ and from $4.7 \%$ to $10.0 \%$, respectively.

Recovery. The recovery for spiked samples obtained by HFLPME-HPLC was calculated by analysing each sample six times under the same concentrations. The recoveries for the actual spiked TCM samples varied from $95.7 \%$ to $99.0 \%$ for Radix angelicae 
sinensis, from $89.7 \%$ to $108.9 \%$ for Radix angelicae dahuricae and from $97.1 \%$ to 105.9\% for Fructus citri sarcodactylis.

\section{Discussion}

\subsection{Bioactive Component Group Screened in TCMs}

The chromatograms of screening and identification of the coumarin and the volatile oil components in three TCMs water decoction performed by using HFCF-HPLC based on SKOV-3, ACHN, HepG-2 cells and HepG-2 cell membrane and organelle are presented in Figure 4. Table 3 list the active components screened and their contents in three TCMs, the target fishing factors (as an index of receptor-drug binding ability in HFCFHPLC), the cell apoptosis rates (the percentage of total apoptotic cells was defined as the sum of early (annexinV-positive, PI-negative cells) and late (annexin- $\mathrm{V}$ positive, PI-positive cells) apoptotic cells) after the medicines acted on the cancer cells determined by flow cytometry analysis. From these results, we can draw the following conclusions. (1) Comparing the retention times with their reference substances, the active components bound with the receptors may be scoparone $\left(t_{\mathrm{C} 2} 6.96\right)$, psoralen $\left(t_{\mathrm{C} 3} 8.50\right)$, bergapten $\left(t_{\mathrm{C} 4} 9.00\right)$, oxypeucedanin $\left(t_{\mathrm{C} 6} 10.02\right)$, imperatorin $\left(t_{\mathrm{C} 7} 12.57\right)$, ligustilide $\left(t_{\mathrm{V} 1}\right.$ 13.98) and isoimperatorin ( $t_{C 8}$ 13.983) Figures 4(B-D). The HPLC-MS results are shown in Figure 4A, the peak $\mathrm{V}_{1}\left(t_{\mathrm{V} 1} 13.98 \mathrm{~min}\right)$ in Radix angelicae sinensis is analyzed by HPLC-MS (Figure 4A(1)). The $\mathrm{m} / \mathrm{z} 191.1$ and $\mathrm{m} / \mathrm{z} 213.07$ are the ions of $[\mathrm{M}+\mathrm{H}]^{+}$ and $[\mathrm{M}+\mathrm{Na}]^{+}$respectively, the exact mass is $190.23836 \mathrm{Da}$, implying the molecular formula of $\mathrm{C}_{12} \mathrm{H}_{14} \mathrm{O}_{2}$. In addition, $\mathrm{C}_{3}\left(\mathrm{t}_{\mathrm{C} 3} 8.50\right.$, the ions of $[\mathrm{M}+\mathrm{H}]^{+}$at $\left.\mathrm{m} / \mathrm{z} 187.44\right), \mathrm{C}_{4}\left(\mathrm{t}_{\mathrm{C} 4}\right.$ 9.00, the ions of $[\mathrm{M}+\mathrm{H}]^{+}$at $\left.\mathrm{m} / \mathrm{z} 217.06\right), \mathrm{C}_{6}\left(\mathrm{t}_{\mathrm{C} 6} 10.02\right.$, the ions of $[\mathrm{M}+\mathrm{H}]^{+}$, $\left[\mathrm{M}+\mathrm{H}-\mathrm{C}_{5} \mathrm{H}_{9} \mathrm{O}\right]^{+},[\mathrm{M}+\mathrm{Na}]^{+}$and $[\mathrm{M}+\mathrm{K}]^{+}$at $\mathrm{m} / \mathrm{z} 287.11,203.05,309.08$ and 325.08 respectively), $\mathrm{C}_{7}\left(\mathrm{t}_{\mathrm{C} 7} 12.57\right.$, the ions of $[\mathrm{M}+\mathrm{H}]^{+}$and $\left[\mathrm{M}+\mathrm{H}-\mathrm{C}_{5} \mathrm{H}_{8}\right]^{+}$at $\mathrm{m} / \mathrm{z} 271.1$ and 203.1) in Radix angelicae dahuricae are analyzed by HPLC-MS (Figure 4A(2)). In Fructus citri sarcodactylis, $\mathrm{C}_{2}\left(\mathrm{t}_{\mathrm{C} 2} 6.96\right.$, the ions of $[\mathrm{M}+\mathrm{H}]^{+}$at $\left.\mathrm{m} / \mathrm{z} 207.08\right)$ is determined by

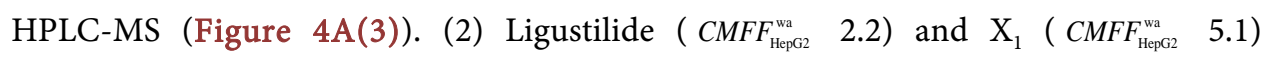
screened by HepG2 cells in Radix angelicae sinensis mainly have effect on its membrane; $\mathrm{Y}_{3}\left(\operatorname{COFF}_{\mathrm{HepC2}}^{\mathrm{wa}} \quad 7.4\right)$ and $\mathrm{Y}_{4}\left(\operatorname{COFF}_{\mathrm{He} C 2}^{\mathrm{wa}} \quad 7.4\right)$ in Radix angelicae dahuricae mainly act on its organelle. However, other active components do weakly or do not act neither on membrane nor organelle. (3) Three TCMs all have strong effect on HepG-2, and the main bioactive components are ligustilide $\left(C F F_{\mathrm{He}(22}^{\mathrm{wa}} 2.3\right)$ and $\mathrm{X}_{1}\left(C F F_{\mathrm{He} C 2}^{\mathrm{wa}} \quad 5.3\right)$ in Radix angelicae sinensis, $\mathrm{Y}_{3}\left(C F F_{\mathrm{He} C 22}^{\mathrm{wa}} 9.0\right), \mathrm{Y}_{4}\left(C F F_{\mathrm{He} C 2}^{\mathrm{wa}} 212.5\right)$, psoralen $\left(C F F_{\mathrm{He} C 22}^{\mathrm{wa}} 4.6\right), \mathrm{Y}_{7}\left(C F F_{\mathrm{He} C 2}^{\text {wa }}\right.$ 5.0), bergapten ( $C F F_{\mathrm{He} C 22}^{\mathrm{wa}} 4.6$ ), oxypeucedanin ( $\left.C F F_{\mathrm{H} p \mathrm{C} 2}^{\mathrm{wa}} 3.1\right)$ in Radix angelicae dahuricae, scoparone ( $C F F_{\mathrm{He} C 22}^{\mathrm{wa}}$ 4.1), $\mathrm{Z}_{3}\left(C F F_{\mathrm{He} C 2}^{\mathrm{wa}} 2.0\right)$ in Fructus citri sarcodactylis. Radix angelicae sinensis have effect on HepG-2 as well as SKOV-3, the active components are ligustilide ( $C F F_{\text {skov }}^{\text {wa }}$ 2.6) and $\mathrm{X}_{1}\left(C F F_{\text {skov }}^{\text {va }}\right.$ 3.4). However, the effect of three TCMs on ACHN are very weak. (4) The trace level of components, such as psoralen $\left(0.028 \mathrm{mg} \cdot \mathrm{g}^{-1}, C F F_{\text {HeC2 }}^{\text {wa }}\right.$ 4.6), bergapten $\left(0.094 \mathrm{mg} \cdot \mathrm{g}^{-1}, C F F_{\mathrm{H} \text { (a) } 2}^{\mathrm{wa}} 4.6\right)$ in Radix angelicae dahuricae, scoparone

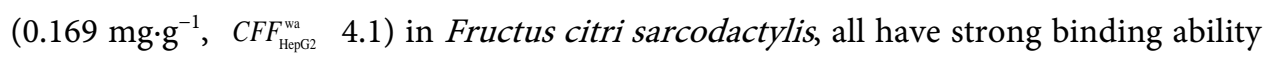
with the cancer cells. (5) Analyzing these cell apoptosis rates, we conclude that the bio- 
active components in Radix angelicae sinensis and Radix angelicae dahuricae can obviously induce SKOV-3 and HepG-2 cells apoptosis. (6)The unidentified active com-

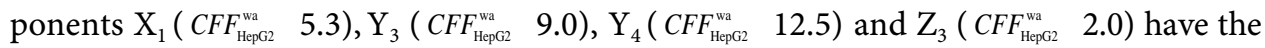
high $C F F$ s.

\subsection{Bioactive Component Content Detected in TCMs}

The proposed HFLPME-HPLC was applied to the simultaneous preconcentration and quantification of coumarin and volatile oil in three medicines. Figure 6 present the chromatograms after pretreatment by using the HFLPME approach, which indicate that the analytes are separated and concentrated effectively by HFLPME. The active component contents detected are listed in the Table 3.

\section{Conclusion}

In this study, we combined HFCF-HPLC with HFLPME-HPLC to simultaneously screen, identify and quantify bioactive components of coumarin and volatile oil in Radix angelicae sinensis, Radix angelicae dahuricae and Fructus citri sarcodactylis. Through the research above, we can see that (1) the characteristics of multi-component and multi-target in three TCMs were confirmed and showed by HFCF-HPLC; (2) isoimperatorin, imperatorin and oxypeucedanin could be responsible for cytotoxicity against SKOV-3 cells, and the experimental result was fairly accorded with the result reported in the literature [22]; (3) many minimal level or even trace level of active components in the TCMs could be critical and important active components with significant therapeutic effects; (4) the unidentified active components with high $C F F$,

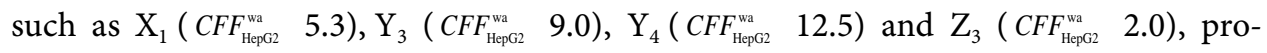
vided us with some good information for finding and researching new anti-cancer active components. In conclusion, HFCF-HPLC, coupled with HFLPME, could be a universal approach to screen and find bioactive components at the cellular level as well as determine their contents (especially the trace level of active components), and research TCM entirety effect of multi-component and multi-target. The approach may provide us a new and good solution to clarify the material basis of anti-cancer effect and conduct
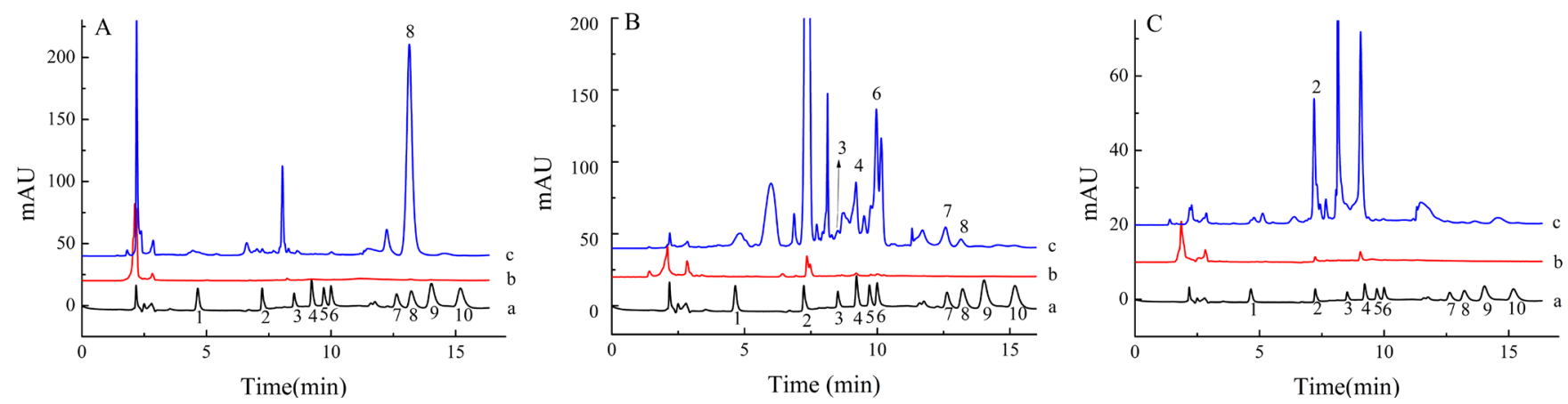

Figure 6. Chromatograms of the coumarins and the volatile oils from Radix angelicae sinensis (A), Radix angelicae dahuricae (B) and Fructus citri sarcodactylis (C) (a) reference substance; (b) before HFLPME; (c) after HFLPME 1. umbelliferone; 2. scoparone; 3. psoralen; 4. bergapten; 5 . isobergapten; 6 . oxypeucedanin; 7. imperatorin; 8 . ligustilide; 9 . isoimperatorin; 10 . columbianadin. 
Table 3. Screening results and contents of the active components in TCMs using HFCF-HPLC couple with HFLPME-HPLC $(\mathrm{n}=3)$.

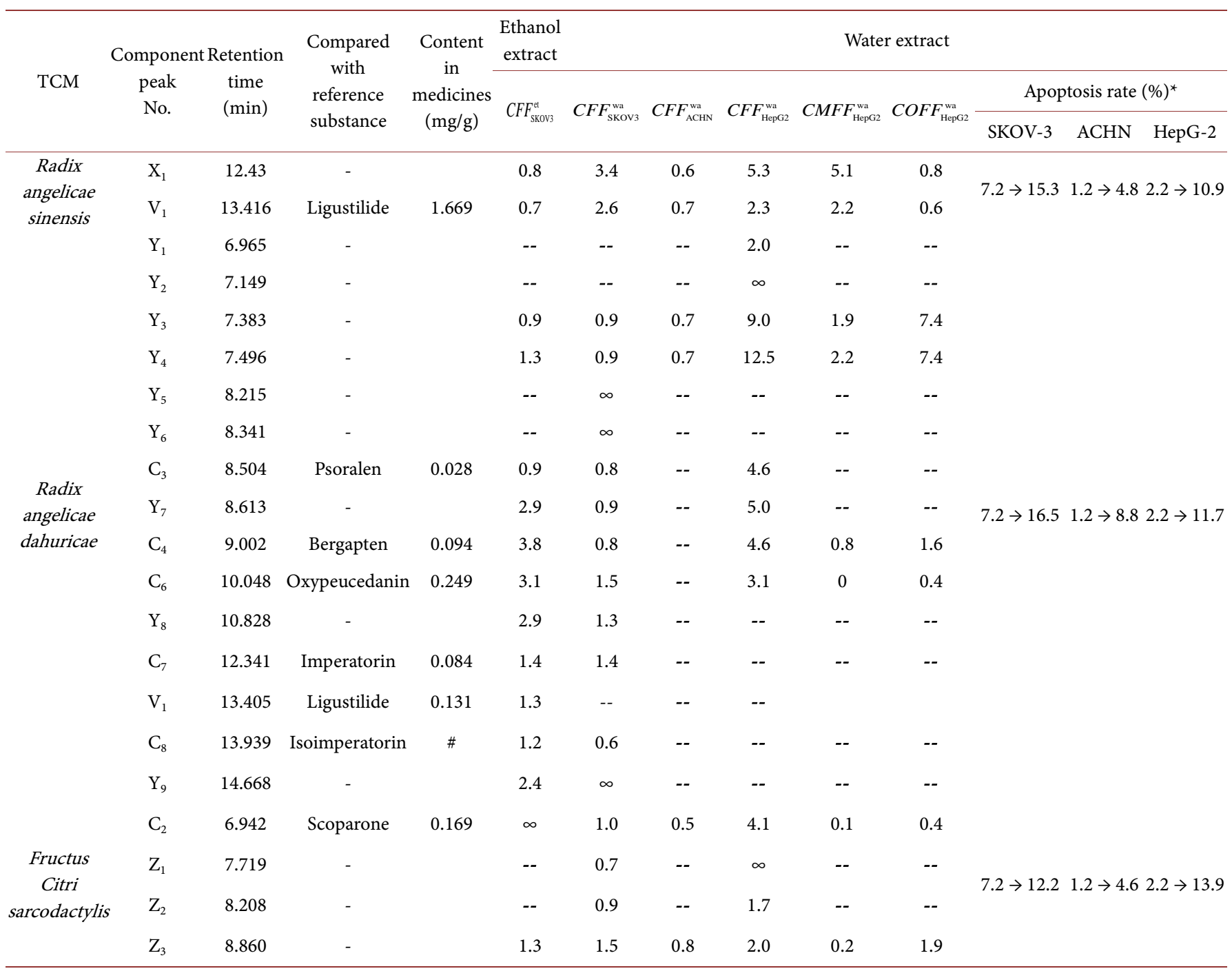

“-” : the active component was unidentified; “--": the active component was not screened out; “\#”: the active component was not detected in the TCMs water decoction; “*”: the apoptosis rate (the percentage of total apoptotic cells was defined as the sum of early (annexinV-positive, PI-negative cells) and late (annexin-V positive, PI-positive cells) apoptotic cells) determined by flow cytometrie before $\rightarrow$ after screening the active components $(\mathrm{P}<0.01)$; " $\infty$ ": the component was screened out by target but not screened out by the nutrient solution or PBS, therefore, the CFF[12] (as an index of receptor-drug binding ability in HFCF-HPLC) of the component closes to infinity, however, it sometimes have no real meaning and do not represent the strong biological activity, for example $\mathrm{Y}_{5}, \mathrm{Y}_{6}, \mathrm{Y}_{9}$ (screened by SKOV-3 cell) and $\mathrm{Y}_{2}$ (screened by HepG-2 cell) in Radix angelicae dahuricae water decoction, $\mathrm{C}_{2}$ (screened by SKOV-3 cell) in Fructus citri sarcodactylis ethanol extract and $\mathrm{Z}_{1}$ (screened by HepG-2 cell) in Fructus citri sarcodactylis water decoction.

personalized quality control for the components associated with efficacy in TCMs.

\section{Acknowledgements}

This study was supported by the National Nature Science Foundation of China (No. 81302734).

\section{Conflict of Interest}

The author confirms that this article content has no conflict of interest. 


\section{References}

[1] Zheng, Y.M., Zheng, S.J., Wang, Y., Lu, A.X. and Shing Ho, W. (2015) Anti-Oxidant and Anti-Cancer Activities of Angelica dahurica extract via Induction of Apoptosis in Colon Cancer Cells. Phytomedicine, 23, 1267-1274. http://dx.doi.org/10.1016/j.phymed.2015.11.008

[2] Wang, X.H., Cheng, K., Han, Y., Zhang, G.Q., Dong, J.L., Cui,Y.Z. and Yang, Z.L. (2016) Effects of Psoralen as an Anti-Tumor Agent in Human Breast Cancer MCF-7/ADR Cells. Biological and Pharmaceutical Bulletin, 39, 815-822.

http://dx.doi.org/10.1248/bpb.b15-00957

[3] Hsieh, M.J., Chen, M.K., Yu, Y.Y., Sheu, G.T. and Chiou, H.L. (2014) Psoralen Reverses Docetaxel-Induced Multidrug Resistance in A549/D16 Human Lung Cancer Cells Lines. Phytomedicine, 21, 970-977. http://dx.doi.org/10.1016/j.phymed.2014.03.008

[4] Su, Z.Y., Khor, T.O., Shu, L., Lee, J.H., Saw, C.L., Wu, T.Y., Huang, Y., Suh, N., Yang, C., Conney, A., Wu, Q. and Kong, A.T. (2013) Epigenetic Reactivation of Nrf2 in Murine Prostate Cancer TRAMP C1 Cells by Natural Phytochemicals Z-Ligustilide and Radix Angelica sinensis via Promoter CpG Demethylation. Chemical Research in Toxicology, 26, 477-485. http://dx.doi.org/10.1021/tx300524p

[5] Zhang, L., Du, J.R., Wang, J., Yu, D.K., Chen, Y.S., He, Y. and Wang, C.Y. (2009) Z-Ligustilide Extracted from Radix Angelica sinensis Decreased Platelet Aggregation Induced by ADP ex Vivo and Arterio-Venous Shunt Thrombosis in Vivo in Rats. Yakugaku Zasshi Journal of the Pharmaceutical Society of Japan, 129, 855-859.

[6] Kurita, K.L., Glassey, E. and Linington, R.G. (2015) Integration of High-Content Screening and Untargeted Metabolomics for Comprehensive Functional Annotation of Natural Product Libraries. Proceedings of the National Academy of Sciences of the United States of America, 112, 11999-12004. http://dx.doi.org/10.1073/pnas.1507743112

[7] Xue, X., Li, L.H., Chen, X., Hu, S. and Bai, X.H. (2013) Hollow Fiber Cell Fishing with High Performance Liquid Chromatography for Screening Bioactive Compounds from Traditional Chinese Medicines. Journal of Chromatography A, 1280, 75-83.

http://dx.doi.org/10.1016/j.chroma.2013.01.033

[8] Yan, Y.Y., Hao, Y.M., Hu, S., Chen, X. and Bai, X.H. (2013) Hollow Fibre Cell Fishing with High Performance Liquid Chromatography for Screening Bioactive Anthraquinones from Traditional Chinese Medicines. Journal of Chromatography A, 1322, 8-17.

http://dx.doi.org/10.1016/j.chroma.2013.10.084

[9] Liu, X., Hu, S., Chen, X. and Bai, X.H. (2014) Hollow Fiber Cell Fishing with HighPerformance Liquid Chromatography for Rapid Screening and Analysis of an Antitumor-Active Protoberberine Alkaloid Group from Coptis chinensis. Journal of Pharmaceutical and Biomedical Analysis, 98, 463-475. http://dx.doi.org/10.1016/j.jpba.2014.06.030

[10] Zhang, L.S., Chen, X., Hu, S. and Bai, X.H. (2014) Rapid Screening of Different Types of Antitumor Compound Groups from Traditional Chinese Medicine by Hollow Fiber Cell Fishing with High Performance Liquid Chromatography. Combinatorial Chemistry \& High Throughput Screening, 17, 827-836. http://dx.doi.org/10.2174/1386207317666141031101709

[11] Zhang, R.X., Chen, X., Hu, S. and Bai, X.H. (2016) Screening and Research of Anti-Cancer Matrine Components Based on Hollow Fiber Cell Fishing with High Performance Liquid Chromatography. Chromatographia, 79, 125-136. http://dx.doi.org/10.1007/s10337-015-3006-8

[12] Li, S., Chen, X., Hu, S., Yan, H.H. and Bai, X.H. (2015) Screening of Bioactive Compounds 
and Research of Possible Targets Based on Hollow Fiber Cell Fishing with High Performance Liquid Chromatography. Analytical Methods, 7, 3124-3133. http://dx.doi.org/10.1039/C5AY00066A

[13] Yan, Y.Y., Chen, X., Hu, S. and Bai, X.H. (2014) Applications of Liquid-Phase Microextraction Techniques in Natural Product Analysis: A Review. Journal of chromatography A, 1368, 1-17. http://dx.doi.org/10.1016/j.chroma.2014.09.068

[14] Megersa, N. (2015) Hollow Fiber-Liquid Phase Microextraction for Trace Enrichment of the Residues of Atrazine and Its Major Degradation Products from Environmental Water and Human Urine Samples. Analytical Methods, 7, 9940-9948. http://dx.doi.org/10.1039/C5AY01927C

[15] Saraji, M., Khayamian, T., Hashemian, Z., Aslipashaki, S.N. and Talebi, M. (2013) Determination of Artemisinin in Artemisia Species by Hollow Fiber-Based Liquid-Phase Microextraction and Electrospray Ionization-Ion Mobility Spectrometry. Analytical Methods, 5, 4190-4195. http://dx.doi.org/10.1039/c3ay40701b

[16] Tian, J., Chen, X. and Bai, X.H. (2012) Analysis of Emodin and Its Metabolites Based on Hollow Fibre Liquid Phase Microextraction. Chinese Journal of Chromatography, 30, 507514. http://dx.doi.org/10.3724/SP.J.1123.2011.12001

[17] Hu, J. and Feng, L.L. (2005) Study on the Determination and Extraction of Ligustilide in Radix Angelicae sinensis and Rhizoma Chuanxiong. Chin. Journal of Shengyang Pharmaceutical University, 22, 145-148.

[18] Li, L., Li, Z.L., He, Y.X. and Yuan, Y. (2008) Study on Extraction Process of Radix Angelicae dahuricae. Lishizhen Medicine and Material Medica Research, 19, 374-375.

[19] Li, W.J. (2013) Extraction and Separation of Effective Components from Bergamot and Their Biological Activities Study in Vitro. Master Thesis, Chongqing University, Chongqing.

[20] Xing, R.R., Chen, X., Hu, S. and Bai, X.H. (2014) Novel Microporous Membrane/Solvent Microextraction for Preconcentration of Cinnamic Acid Derivatives in Rhizoma typhonii. Chromatographia, 77, 553-559. http://dx.doi.org/10.1007/s10337-014-2635-7

[21] Li, S., Hu, S., Chen, X., Bai, X.H. and Zhang, S.Q. (2015) Two-Phase/Three-Phase Hollow Fibre Liquid-Phase Simultaneous Microextraction Combined with HPLC for Analysis of Phenolic Acids and Flavonoids in Traditional Chinese Medicine. Chromatographia, 78, 1159-1167. http://dx.doi.org/10.1007/s10337-015-2930-y

[22] Kim, Y.K., Kim, Y.S. and Ryu, S.Y. (2007) Antiproliferative Effect of Furanocoumarins from the Root of Angelica dahurica on Cultured Human Tumor Cell Lines. Phytotherapy Research, 21, 288-290. http://dx.doi.org/10.1002/ptr.2043 
Submit or recommend next manuscript to SCIRP and we will provide best service for you:

Accepting pre-submission inquiries through Email, Facebook, LinkedIn, Twitter, etc. A wide selection of journals (inclusive of 9 subjects, more than 200 journals)

Providing 24-hour high-quality service

User-friendly online submission system

Fair and swift peer-review system

Efficient typesetting and proofreading procedure

Display of the result of downloads and visits, as well as the number of cited articles

Maximum dissemination of your research work

Submit your manuscript at: http://papersubmission.scirp.org/

Or contact ajac@scirp.org 